\title{
Amiloidosi da transtiretina (ATTR): l'altra faccia della medaglia
}

\author{
Federico Perfetto', Francesco Cappelli², Silvia Farsetti', \\ Elio Dimarcantonio ${ }^{3}$, Silvia Casagrande ${ }^{4}$, Massimo Di Gioia', \\ Marco Di Girolamo ${ }^{5}$ e Franco Bergesio'
}

\begin{abstract}
TTR amyloidosis: the other side of the coin

Transthyretin amyloidosis (ATTR) is becoming an emerging clinical entity, and is currently the most common form of systemic amyloidosis. ATTR consists of two distinct diseases depending on whether the amyloid fibrils derive from the intact molecule of TTR (ATTR wild-type or senile systemic amyloidosis) or from different mutations occurred in the TTR molecule gene (ATTRm). Total-body scintigraphy with diphosphonates has greatly improved the diagnosis in patients with isolated cardiac involvement for both ATTRm and ATTRwt, thus avoiding the need of endomiocardial biopsy in cases without serum and/or urinary monoclonal component (MC). Heart failure alone, or associated with peripheral and autonomic neuropathy, are the main clinical symptoms in these patients. Particular attention must be paid in order to exclude hypertrophic cardiomiopathy or light chain $(A L)$ forms in patients with $M C$. Today, besides hepatic transplantation, which is almost reserved to patients with early Val30Met neuropathy, many new therapeutic alternatives can be offered to these patients. Tafamidis, a TTR-stabilizer that has recently proved to be effective in cardiac forms of both ATTRm and ATTRwt, is now ready for clinical use. In addition, drugs silencing the TTR messenger RNA should soon be available for treatment. The many therapeutic opportunities available today for ATTR, strenghten even more the need for an early diagnosis of the disease both in ATTRm and ATTRwt.
\end{abstract}

\section{Keywords}

Transtyretin, Hereditary amyloidosis, Senile amyloidosis, Infiltrative cardiomyopathy

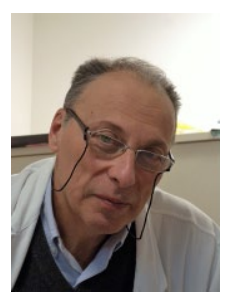

\section{Introduzione}

Un paziente maschio in età adultal avanzata si rivolge al proprio medico o cardiologo di fiducia per la recente insorgenza di dispnea da sforzo. Una visita cardiologica con ecografia del cuore rivela uno spiccato aumento dello spessore della parete e del setto del ventricolo sinistro con alterazioni diastoliche evidenti ma con una frazione di eiezione ancora conservata. L'ECG mostra dei voltaggi inaspettatamente ridotti e comunque non adeguati rispetto al quadro di ipertrofia. All'anamnesi si rivela una storia di ipertensione arteriosa ben controllata e il riscontro di una pregressa patologia del tunnel carpale bilaterale. Non vi sono altri segni o sintomi clinici di rilievo.

È questo il quadro di gran lunga più comune ( $>90 \%$ dei casi) con cui oggi si presenta il paziente affetto da Amiloidosi da Transtiretina (ATTR) in Toscana, mentre rappresenta soltanto il $20 \%$ circa dei casi clinici di ATTR all'esordio nel nostro paese e nel mondo ${ }^{1}$ (www. thaos.net).

In altri paesi come il Portogallo, il Brasile, la Svezia e il Giappone, dove la malattia si presenta in modo endemico, il quadro d'esordio è diverso e colpisce soggetti più giovani con i sintomi di una polineuropatia prevalentemente

\footnotetext{
'Centro di Riferimento Regionale per le Amiloidosi, AOU Careggi, Firenze, Italy

2Divisione di Cardiologia Interventistica Strutturale AOU Careggi, Firenze, Italy

3SOD Nefrologia e Dialisi, AOU Careggi, Firenze, Italy

4SOD Neurologia 2, AOU Careggi, Firenze, Italy

${ }^{5}$ Coordinatore Centro Amiloidosi Ospedale Fatebenefratelli "S.

Giovanni Calabita", Roma
}

\section{Corrispondenza:}

Federico Perfetto, Centro di Riferimento Regionale per le Amiloidosi, Azienda Ospedaliero-Universitaria Careggi, Largo Brambilla, Firenze Italy. Email: perfetto@unifi.it 
sensitiva con coinvolgimento autonomico anche isolato (25\%-30\%) o associata ad una cardiopatia infiltrativa $(50 \%)^{2}$ (www.thaos.net). In queste regioni è nota da tempo una base eredofamiliare della malattia con una trasmissione di tipo autosomico dominante.

Quadri così paucisintomatici e aspecifici possono essere facilmente confusi con quelli di una cardiopatia ipertrofica o ipertensiva o con quelli di neuropatie dismetaboliche $o$ autoimmuni ad andamento subacuto/cronico. La diagnosi di amiloidosi quindi in questi pazienti rischia di essere misconosciuta per lungo tempo con evidenti ricadute prognostiche e terapeutiche.

Il ritardo diagnostico si aggira sui quattro anni per le forme neuropatiche, mentre può arrivare fino ad otto per quelle cardiache. ${ }^{3}$

Inoltre, una volta posta correttamente la diagnosi di amiloidosi, la sua caratterizzazione come una forma da catene leggere $(\mathrm{AL})$ o da transtiretina (TTR) non è fattibile sulla sola base delle manifestazioni cliniche. Questo accade perché il coinvolgimento cardiaco e/o neurologico è molto simile nelle due forme, e la presenza, non infrequente, di una componente monoclonale (CM) incidentale nelle forme di ATTR rende la diagnosi più complicata, con il rischio di scambiare una forma AL per una ATTR con conseguenze prognostiche e terapeutiche disastrose per il paziente. ${ }^{4}$

\section{La molecola della transtiretina}

La transtiretina, nota in passato anche come prealbumina, è costituita da un tetramero composto da quattro monomeri legati assieme, ciascuno di 127 aminoacidi, e sintetizzati principalmente dal fegato, ma in minime quantità anche dall'epitelio pigmentoso della retina e dai plessi coroidei cerebrali. Fisiologicamente ha la funzione di trasportare nel siero la molecola della tiroxina e del retinolo (vit A). ${ }^{2}$

In corso di mutazioni generalmente puntiformi ("missense") del gene della TTR, la semplice sostituzione di un aminoacido con un altro nella catena polipeptica, determina la destabilizzazione dell'intero tetramero che facilmente provoca il rilascio dei monomeri che, non più uniti tra di loro, tendono a dispiegarsi ("misfolding") perdendo la loro configurazione molecolare nativa e dando luogo ad oligomeri instabili che successivamente si aggregano in fibrille amiloidi che poi si depositano nei tessuti.

Tuttavia, la molecola della TTR è una proteina nativamente instabile in cui le catene polipeptidiche dei monomeri che la costituiscono tendono spontaneamente ad andare incontro a "misfolding". Tali catene, con il passare degli anni, subiscono modificazioni proteolitiche che le rendono particolarmente instabili e suscettibili a trasformarsi in fibrille che poi si depositano nel cuore di individui maschi di età avanzata. Nell'ambito delle amiloidosi da transtiretina possiamo dunque distinguere due forme: 1) forme mutate o ereditarie (ATTRm) e 2) forme non mutate o wild-type (wt, ATTRwt) o senili.

\section{Epidemiologia dell'ATTR}

È da sempre nota l'esistenza di forme di amiloidosi ereditaria, tra cui quella di gran lunga più comune è quella legata a mutazioni della molecola della TTR, di cui oggi si conoscono oltre 100 varianti amiloidogeniche (ATTRm). Si sapeva tuttavia che anche la molecola intatta o wt poteva dar luogo alla formazione di fibrille amiloidi con interessamento pressochè esclusivo del cuore. Questa forma, tipica degli anziani di sesso maschile, era anche conosciuta come amiloidosi senile sistemica. ${ }^{2}$ Di questa forma non si conosce tuttora la reale incidenza, ma studi autoptici avrebbero dimostrato la presenza di depositi di amiloidosi cardiaca, anche se di modeste entità, in oltre il $25 \%$ degli individui di età $>80$ anni. $^{5}$

Il motivo di ciò risiede nel fatto che la diagnosi istopatologica di ATTRwt è resa molto difficile per la sensibilità assai scarsa del prelievo di tessuto adiposo periombelicale in questi pazienti ( $15 \%$-20\% circa). Perciò la diagnosi ha comportato necessariamente il ricorso ad una biopsia endomiocardiaca, spesso tuttavia difficilmente praticabile per l'età avanzata e le comorbidità presenti in questi pazienti. Questo ha determinato, fino a pochi anni fa, un notevole ostacolo nella diagnosi delle forme wt.

Per fortuna, da alcuni anni, è venuta in nostro soccorso una metodica diagnostica semplice, poco costosa e alla portata di tutti gli ospedali: la scintigrafia ossea con bifosfonati (99mTc-DPD/HMDP/PYP). Questa tecnica ha permesso di distinguere con sicurezza e nessuna invasività le forme cardiache da TTR (sia mutata che wt che mostrano una patognomonica captazione cardiaca del tracciante osseo), da quelle AL (poco o per nulla captanti), evitando nella maggior parte dei casi il ricorso all'indagine bioptica. ${ }^{5}$ Questo ha facilitato e velocizzato la diagnosi di amiloidosi cardica da TTR consentendo di avere un quadro epidemiologico più preciso dell'incidenza e prevalenza delle due forme di ATTR, di cui le wt rappresentano oggi la forma di amiloidosi di gran lunga più diagnosticata (figura 1).

Da circa 10 anni è stato costituito un Registro internazionale per l'osservazione e il monitoraggio dell'evoluzione clinica di tutte le forme di amiloidosi da TTR, sia mutate che non. Il registro rappresenta il migliore strumento per lo studio della relativa frequenza, diffusione e manifestazioni cliniche di queste forme (www.thaos.net).

\section{Amiloidosi da transtiretina mutata (ATTRm)}

Oggi si conoscono più di 100 mutazioni puntiformi che determinano cioè la sostituzione di singoli residui aminoacidici nella molecola della TTR. La maggior parte 
si associano allo sviluppo della malattia e rendono ragione dell'ampia variabilità fenotipica i cui principali determinanti, oltre al tipo di mutazione, sono la regione geografica di appartenenza, l'età e il sesso del paziente.,2,6

La maggior parte dei pazienti sono eterozigoti ed esprimono sia la molecola della TTR intatta che quella mutata. Le forme mutate coprono $>80 \%$ di tutte le forme di ATTR registrate nel Thaos (www.thaos.net). Nell'ambito della più comune di esse, ovvero la Val30Met, il fenotipo varia anche a seconda che la malattia presenti una distribuzione endemica, come avviene in alcune parti del Portogallo, Giappone e nord della Svezia, oppure non endemica (sporadica). Il fenotipo della forma Val30Met endemica si manifesta tipicamente con una polineuropatia

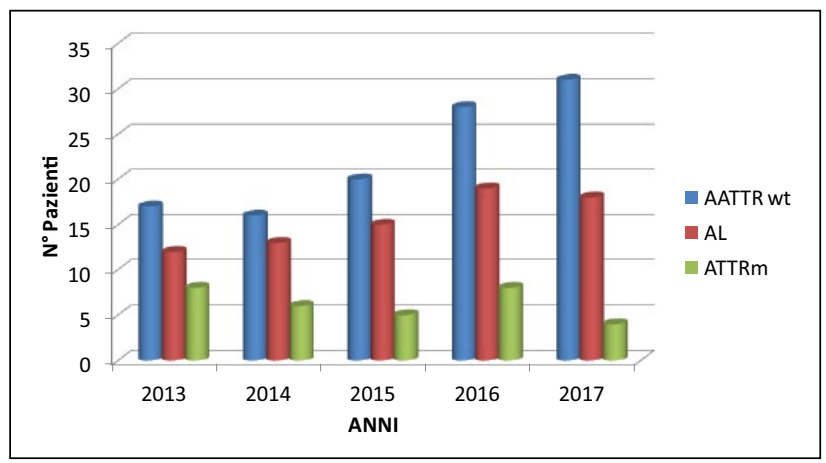

Figura I. Incidenza dell'amiloidosi da transtiretina (wt e mutata) vs quella dell'AL presso il Centro di RiferimentoToscano per le Amiloidosi (2013-20I7). sensitivo-motoria con associata neuropatia autonomica che esordisce in età giovanile adulta (30-40 anni). Il coinvolgimento cardiaco è raro e tardivo e coinvolge spesso il sistema di conduzione del cuore. Maggiore variabilità fenotipica hanno le forme non endemiche che mostrano un esordio più tardivo e un più frequente coinvolgimento cardiaco con scompenso congestizio. ${ }^{6}$ Questa mutazione rappresenta da sola il $70 \%$ di tutte le forme mutate. Nel rimanente $30 \%$ dei casi, si concentrano tutte le altre mutazioni responsabili sia di forme esclusivamente cardiologiche $(37 \%)$ che neurologiche o miste (63\%). In uno studio multicentrico italiano di 186 pazienti con differenti mutazioni di TTR, il $17 \%$ presentavano un fenotipo esclusivamente cardiologico di cui la mutazione responsabile più frequente è risultata la Ile68Leu, mentre il 58\% presentavano un fenotipo misto e il $25 \%$ uno neurologico. ${ }^{1}$ Nonostante la numerosità delle mutazioni, è possibile ricondurre sostanzialmente a tre $\mathrm{i}$ fenotipi più comuni: uno neurologico, caratterizzato da un quadro di polineuropatia periferica distale di cui la Val30Met rappresenta il prototipo (FAP: famylial amyloid polyneuropathy); uno cardiologico, con interessamento più o meno esclusivo del cuore (FAC: famylial amyloid cardiomiopathy) e di cui la la Val122Ile e la Ile68Leu sono le forme più comuni; ed infine uno misto, con interessamento neurologico e cardiaco variabile., ${ }^{2,6}$

Va inoltre ricordato come possa esistere una variabilità fenotipica anche all'interno delle singole mutazioni. ${ }^{3}$ A ciascun fenotipo corrispondono genotipi ben precisi, come è illustrato nella figura 2 , dove sono anche evidenziati

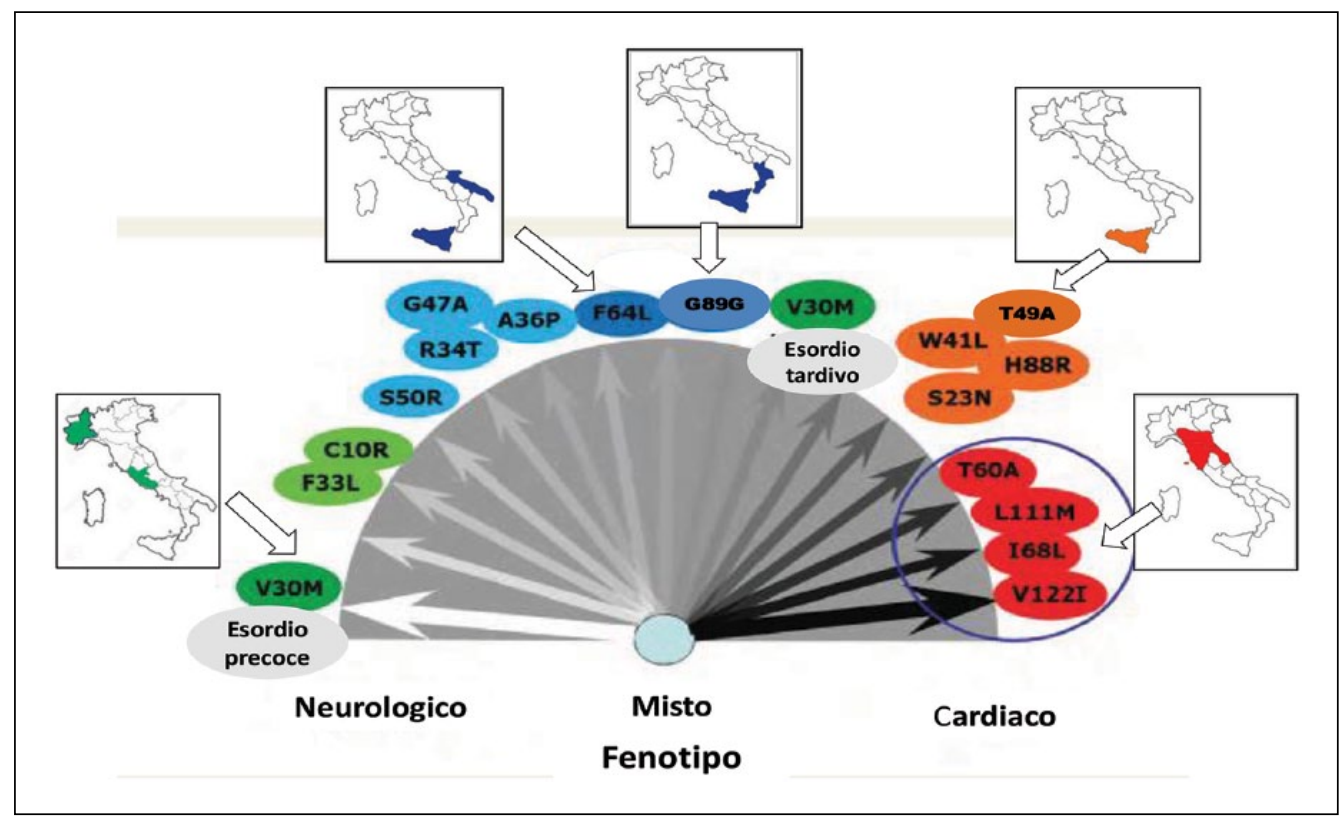

Figura 2. Ventaglio delle possibili correlazioni genotipo-fenotipo nell'amiloidosi familiare (ATTRm): da un fenotipo quasi esclusivamente neurologico ad uno prevalentemente, se non esclusivamente, cardiologico, con in mezzo fenotipi "misti” con grado variabile di interessamento neurologico e cardiaco. 
quelli più comuni riscontrati nel nostro Paese, con le rispettive aree geografiche dove risultano prevalenti.

In particolare, la variante Glu89Gln, caratterizzata da una forma prevalentemente neuropatica, è prevalente in Sicilia nel territorio di Noto e Avola, mentre la mutazione Val30Met è presente in un cluster di famiglie della provincia di Cuneo e nel Lazio. Recentemente è stata segnalata un'area endemica per la presenza della variante Ile68Leu nella regione appenninica a cavallo tra EmiliaRomagna e Toscana. ${ }^{1}$ Questa mutazione, ad espressione fenotipica cardiologica pura, rappresenta oltre la metà delle forme mutate nella regione Toscana seguita sorprendentemente in ordine di frequenza dalla Val122Ile, anch'essa una forma cardiologica pura, tradizionalmente considerata una forma tipica della popolazione AfroAmericana-Caraibica. ${ }^{2,6}$ Per tutte queste varianti è ragionevole ipotizzare un effetto fondatore.

A tutt'oggi purtroppo non esiste un registro nazionale delle forme mutate di ATTR che ci possa fornire dati precisi su prevalenza e distribuzione geografica delle differenti mutazioni presenti nel nostro Paese.

\section{Amiloidosi da transtiretina wild-type (ATTRwt o senile)}

La forma ATTRwt è molto più comune nei maschi, con un rapporto maschi:femmine di circa 9:1, e può comparire già dopo i 60 anni, ma diventa più frequente dopo gli 80 . Nonostante sia definita come forma sistemica, il cuore è generalmente l'unico organo interessato, insieme alla regione carpale sotto forma della sindrome del tunnel carpale $^{2,6}$ che spesso precede anche di molti anni la sintomatologia cardiaca. Recentemente è stato evidenziato talora anche un interessamento del tessuto muscolare periferico e del polmone.

Ad oggi questi casi rappresentano solo il $14 \%$ di tutti quelli registrati nel Thaos (www.thaos.net).

Questo dato sembra molto sottostimato alla luce anche della crescente incidenza osservata per questa forma negli ultimi anni, come dimostrano anche i dati della nostra casistica dove l'amiloidosi senile comprende attualmente oltre la metà di tutte le diagnosi di amiloidosi formulate in un anno, e rappresenta quindi in assoluto la forma di amiloidosi con più alta incidenza (figura 1).

Che la malattia sia comunemente sottostimata emerge anche da uno studio su pazienti di età $>60$ anni, affetti da scompenso cardiaco con frazione di eiezione conservata (HFpEF), in cui l'amiloidosi senile è stata diagnosticata nel $13 \%$ dei casi. ${ }^{7}$

Le manifestazioni cliniche all'esordio sono simili a quelle presentate dai pazienti con le forme familiari ad interessamento prevalentemente cardiologico: scompenso cardiaco a lenta evoluzione in assenza di dilatazione ventricolare sinistro, senza o con lieve deficit della frazione di eiezione del vetricolo sinistro.

\section{Manifestazioni neurologiche}

La manifestazione neurologica più comune è la sindrome del tunnel carpale, spesso bilaterale, talvolta sincrona e quasi inevitabilmente primo sintomo d'esordio della patologia. La seconda manifestazione più frequente è la neuropatia periferica lunghezza-dipendente.

Essa si presenta, pur all'interno di un'ampia variabilità fenotipica, come la manifestazione più comune della maggior parte delle forme mutate (circa $3 / 4$ di tutti i casi).

Tipicamente si manifesta con un iniziale interessamento delle piccole fibre, prima delle estremità distali degli arti inferiori, ed in seguito dei superiori con evoluzione centripeta.

Il paziente inizialmente presenta parestesie dolorose, ipoestesia prevalentemente termica e segni e sintomi di coinvolgimento autonomico. Il coinvolgimento delle grosse fibre sensitive e motorie avviene solamente in un secondo momento e si presenta con una riduzione della sensibilità tattile, con conseguente atassia sensitiva determinante difficoltà nella deambulazione $\mathrm{e}$ nell'esecuzione dei movimenti fini delle mani cui si associa un progressivo deficit di forza fino alla totale paralisi.

La neuropatia autonomica è spesso la manifestazione più invalidante. Inizialmente limitata al versante cardiovascolare con i segni e sintomi dell'ipotensione ortostatica, coinvolge con il passare del tempo tutti gli altri organi e sistemi determinando impotenza da disfunzione erettile nel maschio, importanti alterazioni della motilità intestinale che si manifestano con stipsi ostinata alternata a diarrea, nausea, vomito, dispepsia e marcato calo ponderale (spesso primo sintomo), ritenzione $\mathrm{e} / \mathrm{o}$ incontinenza urinaria, disidratazione e alterazioni trofiche della cute fino alla comparsa di vere e proprie ulcere a livello delle estremità distali. ${ }^{2,6}$

L'interessamento neurologico è presente in oltre la metà dei pazienti con ATTRm, nei quali la mutazione Val30Met è responsabile dei $3 / 4$ dei casi, la metà dei quali in Portogallo (www.thaos.net).

Evenienza rara, e solo nel corso di alcune specifiche mutazioni, è l'interessamento dei vasi leptomeningei, interessamento che determina manifestazioni aspecifiche come ictus ischemico o emorragico, emorragie subaracnoidee, decadimento cognitivo e quadri comiziali. ${ }^{6}$

\section{Manifestazioni cardiache}

Le manifestazioni cardiache sono presenti in circa la metà dei pazienti con ATTR. Di questi, il 57\% è dovuto a forme wt, mentre il restante $43 \%$ alle forme mutate.

Alcune varianti presentano un interessamento cardiaco esclusivo, di cui la Val122Ile e la Ile68leu sono le più comuni e le piu gravi dal punto di vista prognostico. Diversamente, la Val30Met, in cui il disturbo cardiaco concomita con quello neurologico, sembra influenzare 
Tabella I. Principali caratteristiche cliniche, ECG ed ecocardiografiche dei pazienti affetti da ATTR (mutata e wt) rispetto ai pazienti con AL e CMPI.

\begin{tabular}{|c|c|c|c|c|}
\hline & ATTRm & ATTRwt & $\mathbf{A L}$ & CMPI \\
\hline Età media (anni) & 70.2 & 79.5 & 69.5 & 64 \\
\hline Maschi (\%) & $70 \%$ & $89 \%$ & $58 \%$ & $87 \%$ \\
\hline Spessore VSx & $\begin{array}{l}\text { Moderatamente } \\
\text { aumentato }\end{array}$ & Molto aumentato & Lievemente aumentato & Molto aumentato \\
\hline Ipertrofia VSx & $\begin{array}{l}\text { Tendenzialmente } \\
\text { simmetrica }\end{array}$ & $\begin{array}{l}\text { Tendenzialmente } \\
\text { simmetrica }\end{array}$ & $\begin{array}{l}\text { Tendenzialmente } \\
\text { simmetrica }\end{array}$ & Variabile \\
\hline $\mathrm{FE}$ & $\begin{array}{l}\text { Lievemente } \\
\text { ridotta }\end{array}$ & $\begin{array}{l}\text { Moderatamente/ } \\
\text { sostanzialmente ridotta }\end{array}$ & Normale o poco ridotta & Normale \\
\hline Disfunzione diastolica & Frequente & Frequente & Frequente & Frequente \\
\hline Bassi voltaggi & $<25 \%$ dei casi & $<25 \%$ dei casi & Frequente & Estremamente raro \\
\hline Fibrillazione atriale & $44 \%$ & $37 \%$ & $10 \%$ & $7 \%$ \\
\hline Versamento pericardico & Frequente & Frequente & Frequente & Estremamente raro \\
\hline Ispessimento valvolare & Frequente & Frequente & Frequente & Assente \\
\hline $\begin{array}{l}\text { Captazione miocardica } \\
\text { dei bifosfonati }\end{array}$ & Forte & Forte & Debole/assente & Assente \\
\hline $\begin{array}{l}\text { Valori NT-proBNP e } \\
\text { troponina cardiaca }\end{array}$ & $\begin{array}{l}\text { Moderatamente } \\
\text { aumentati }\end{array}$ & Severamente aumentati & $\begin{array}{l}\text { Moderatamente } \\
\text { aumentati }\end{array}$ & $\begin{array}{l}\text { Moderatamente } \\
\text { aumentati }\end{array}$ \\
\hline $\begin{array}{l}\text { Sindrome del tunnel } \\
\text { carpale }\end{array}$ & $\begin{array}{l}\text { Frequente } \\
\text { bilaterale }\end{array}$ & Frequente bilaterale & Possibile bilaterale & Non associata \\
\hline Organi coinvolti & $\begin{array}{l}\text { Cuore/SNP/SNA/ } \\
\text { occhio, rene }\end{array}$ & $\begin{array}{l}\text { Cuore, più raro } \\
\text { polmone e muscoli }\end{array}$ & $\begin{array}{l}\text { Cuore, Rene, SNP/SNA, } \\
\text { fegato, milza, tratto GI }\end{array}$ & Non associati \\
\hline
\end{tabular}

VSx= ventricolo sinistro; $\mathrm{CMPI}=$ cardiomiopatie ipertrofiche; $\mathrm{FE}=$ frazione d'eiezione

molto meno la qualità della vita e la prognosi, interessando principalmente, soprattutto nelle forme endemiche, il tessuto di conduzione con blocchi fascicolari o raramente blocchi atrioventricolari (AV) avanzati con necessità di impianto di pace maker.

Nelle forme con coinvolgimento cardiaco più importante, i depositi di amiloide possono localizzarsi in tutti i tessuti cardiaci, dal tessuto di conduzione al miocardio atriale e ventricolare, dal tessuto valvolare ai vasi coronarici e al pericardio. L'infiltrazione miocardica comporta il progressivo incremento degli spessori ventricolari sinistro e destro e del setto interatriale. Nella metà dei pazienti si osserva un pattern di riempimento diastolico restrittivo, anche se un terzo di loro non mostra segni di difettosa funzione diastolica. La funzione ventricolare sinistra è di solito conservata o lievemente ridotta. Gli atri appaiono ingranditi, spesso fibrillanti e con apposizioni trombotiche al loro interno. I ventricoli, coerentemente col processo infiltrativo, sono piccoli. L'infiltrazione delle valvole è responsabile di gradi variabili di insufficienza valvolare. Tutte queste alterazioni sono ben visibili ad uno studio ecocardiografico che mostra anche una tipica ecogenicità brillante ("sparkling") delle pareti ventricolari infiltrate. La clinica spazia da asintomatici blocchi AV o di branca fino a, più spesso, quadri di scompenso cardiaco per lo più destro caratterizzati da edemi declivi importanti, epatomegalia, turgore giugulare e ascite, che in tempi variabili evolve verso un'insufficienza cardiaca severa e progressiva. .,3,6 $^{2,6}$
Nelle forme wt l'interessamento si manifesta con un quadro variabile di scompenso con aspetti ecocardiografici sovrapponibili a quelli osservati in altre forme di amiloidosi (forme mutate e AL) anche se questi pazienti mostrano all'esordio un incremento maggiore dello spessore medio del ventricolo sinistro con una minore frazione di eiezione. ${ }^{1,5,6}$

Il quadro ecocardiografico di marcata "ipertrofia" delle ATTR ( sia mutate che wt) contrasta spesso con il quadro elettrocardiografico di normali voltaggi nelle derivazioni periferiche, dove non si osserva tuttavia il quadro di bassi voltaggi più caratteristico delle forme AL.

Nella tabella 1 sono riportati i principali dati clinici e cardiologici delle forme mutate e di quelle wt a confronto con quelli delle forme $\mathrm{AL}$ e delle cardiomiopatie ipertrofiche (CMPI) con cui possono venire più comunemente confuse.

I pazienti affetti da ATTR con interessamento cardiaco hanno caratteristiche cliniche diverse da quelli che presentano un fenotipo misto. Nello specifico, l'età di insorgenza è mediamente $>65$ anni (più alta mediamente di 17 anni), la prevalenza è nettamente superiore nei soggetti di sesso maschile e l'interessamento cardiaco è più pronunciato anche rispetto alle forme $\mathrm{AL}$, come evidenziato dai dati ECG ed ecocardiografici (tabella 1).

A parte l'età, solo l'esame genetico ci può aiutare a distinguere le forme mutate dalle quelle wt, non essendoci nessuna significativa differenza tra i reperti elettrocardiografici ed ecocardiografici delle due forme. ${ }^{6,8}$ 
La maggiore prevalenza del sesso maschile nelle forme ad esclusivo coinvolgimento cardiaco ha suggerito l'esistenza di un effetto protettivo del sesso femminile nei confronti del coinvolgimento cardiaco. ${ }^{6} \mathrm{La}$ malattia presenta generalmente un decorso lentamente progressivo che si giova della terapia convenzionale contro lo scompenso cardiaco.

\section{Altre manifestazioni cliniche}

L'occhio viene interessato da opacità del vitreo che causano progressiva perdita del visus con necessità di procedere alla vitrectomia. In rari casi, si ha la cosiddetta "pupilla smerlata" ("scalloped") tipica del coinvolgimento ciliare e talora si può osservare un glaucoma. ${ }^{6}$

È stato inoltre descritto un interessamento renale con insufficienza renale e proteinuria in almeno 15 varianti, tra cui la Val30Met è quella più documentata. La nefropatia colpisce i pazienti con le forme neurologiche a esordio tardivo, con bassa penetranza familiare e alterazioni del ritmo cardiaco. ${ }^{9}$ Il coinvolgimento di questi organi può restare per molto tempo asintomatico.

\section{La diagnosi}

\section{II sospetto}

La diagnosi di amiloidosi da ATTR non è facile e, se il clinico non pone l'amiloidosi nel novero delle diagnosi possibili, rischia di non farla o di effettuarla con notevole ritardo. Non raramente i pazienti giungono ad una diagnosi definitiva dopo aver consultato nel corso dell'ultimo anno di malattia più specialisti, neurologi, cardiologi o internisti. Il sospetto diagnostico varia a seconda del sintomo predominante. Se il paziente si presenta al medico con una sintomatologia neurologica periferica, l'amiloidosi deve andare in diagnosi differenziale con tutte le forme di neuropatia carenziale, dismetabolica, autoimmune $\mathrm{o}$ secondarie ad una gammopatia monoclonale. L'eventuale presenza di una familiarità per patologia neurologica $o$ cardiaca (nel caso di un'eventuale forma mista) rafforza molto il sospetto diagnostico, ma purtroppo nelle regioni dove la malattia non è endemica l'anamnesi risulta spesso muta. In questo, la conoscenza della distribuzione geografica dei piccoli focolai italiani di endemia di Val30Met o Glu89G o di Ile68Leu può orientare molto il sospetto diagnostico.

L'eventuale concomitanza di una cardiopatia con ipertrofia marcata delle pareti ventricolari può facilitare la diagnosi nei fenotipi misti in quanto restringe il ventaglio diagnostico. Le maggiori difficoltà riguardano soprattutto i casi ad esclusivo interessamento cardiaco in assenza di una storia di familiarità in cui il quadro clinico e strumentale è suggestivo di una cardiopatia ipertensiva o ipertrofica. Sono questi i casi delle forme senili e delle forme mutate con esclusivo fenotipo cardiologico (figura 2). 2,6,8
L'iter diagnostico di questi pazienti prevede la ricerca anamnestica di una sindrome del tunnel carpale bilaterale o la rottura atraumatica del tendine del capo lungo del bicipite brachiale che costituiscono due vere e proprie "spie" di questa patologia, oppure la presenza di un quadro clinico di scompenso cardiaco con frazione di eiezione conservata,,${ }^{7,8}$ oppure un aumento della massa ventricolare sinistra all'ecocardiogramma non accompagnato da segni elettrocardiografici di ipertrofia ventricolare sinistra ${ }^{6}$ in pazienti con ipertensione arteriosa in assenza di stenosi aortica o cardiomiopatia ipertrofica sarcomerica. In questo caso, l'ECG e l'utilizzo della RMN cardiaca con e senza mezzo di contrasto può essere dirimente nella diagnosi differenziale tra amiloidosi e cardiomiopatia ipertrofica sarcomerica. ${ }^{8}$

Il passo successivo è la conferma del sospetto diagnostico che deve prevedere anche l'identificazione del tipo di amiloidosi sottostante.

Purtroppo, l'aspirato del grasso periombelicale, indagine istologica di riferimento per la diagnosi, risulta meno $\mathrm{o}$ assai poco affidabile in corso di ATTR (sensibilità del $70 \%$ nelle ATTRm e del 15\%-20\% nelle ATTRwt). Perciò, nel caso di un fenotipo neurologico (sospetto di una polineuropatia amiloidotica), se le indagini tradizionali (grasso periombelicale o ghiandole salivari minori) risulteranno negative, dovremo necessariamente procedere alla biopsia del nervo periferico (nervo tibiale anteriore) ben sapendo però che la biopsia indurrà un danno permanente alla fibra nervosa. Una volta ottenuta la conferma bioptica di amiloidosi, dobbiamo identificarne il tipo: AL o ATTRm?

Se si esclude la prima eventualità, per la mancanza di una $\mathrm{CM}$ all'IFX sierica e/o urinaria, si ricercherà la presenza di una mutazione della molecola della TTR mediante analisi sequenziale del gene. L'eventuale presenza di una $\mathrm{CM}$ non è tuttavia sufficiente a porre diagnosi di $\mathrm{AL}$ e ad escludere una forma mutata, data la relativa frequenza di una $\mathrm{CM}$ "incidentale" nei pazienti $>70$ anni per cui è indispensabile in questi casi ricorrere alla tipizzazione tissutale mediante immunoistochimica in microscopia elettronica ("immunogold") o analisi proteomica. $^{2,10}$ Ricordiamo a questo proposito come la tecnica dell'immunoistochimica tradizionale, mediante antisieri rivolti verso la molecola della transtiretina o delle catene leggere presenti nel tessuto bioptico, non sia affidabile e sia stata spesso responsabile di false diagnosi. ${ }^{2}$

Nel caso invece di un fenotipo misto in cui coesistano i sintomi neurologici e cardiologici o di un fenotipo esclusivamente cardiologico, non è oggi più necessario ottenere una conferma istologica mediante biopsia del nervo o del cuore.

Infatti, l'impiego della scintigrafia con bifosfonati (99mTc-DPD /HMD /PYP) si è dimostrato un metodo sicuro ed affidabile per la diagnosi di tutte le forme cardiache di ATTR (siano esse mutate che wt) indipendentemente dalla conferma bioptica, purchè l'intensità della captazione 


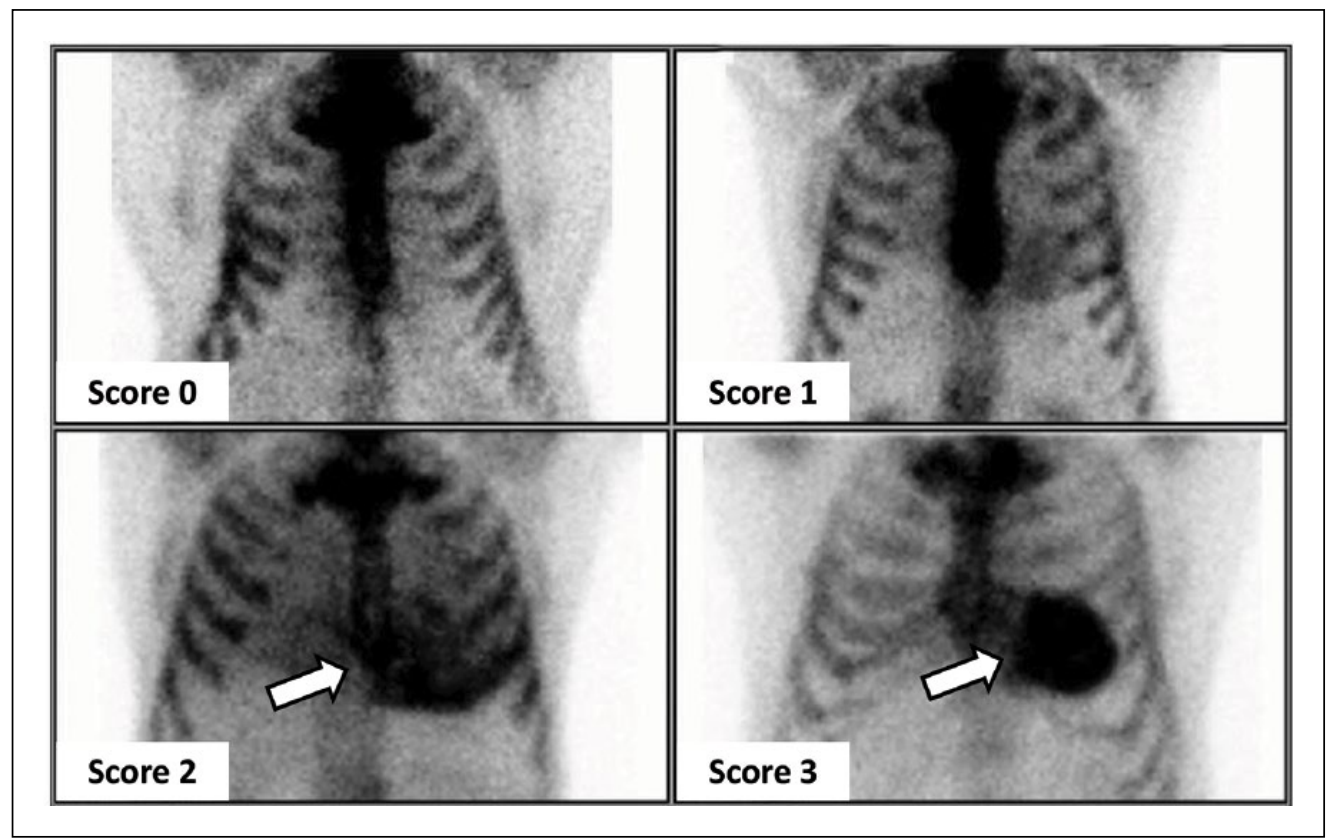

Figura 3. Scintigrafia con difosfonato (99mTc-HMDP) per lo studio della captazione cardiaca in corso di amiloidosi da TTR. Valutazione semiquantitativa mediante il punteggio visivo di Perugini*.

Score 0: nessuna captazione cardiaca in presenza di normale captazione ossea; Score I: debole captazione cardiaca, inferiore a quella ossea; Score 2: moderata captazione cardiaca con captazione ossea attenuata; Score 3: elevata captazione cardiaca con ridotta o assente captazione ossea. *Perugini E, Guidalotti PL, Salvi F, Cooke RM, et al. Noninvasive etiologic diagnosis of cardiac amyloidosis using 99mTc-3,3-diphosphono-I,2propanodicarboxylic acid scintigraphy. J Am Coll Cardiol 2005; 46(6): 1076-1084.

cardiaca del tracciante sia uguale o superiore a quella dell'osso (grado $2^{\circ}$ o $3^{\circ}$ della scala di Perugini che prevede un grado 0 se la captazione cardiaca è assente, e un grado 1 se la captazione è debole e inferiore a quella dell'osso) e non vi sia la contemporanea presenza di una $\mathrm{CM}^{5}$ (figura 3). In questa eventualità (circa il 15\% dei casi), per la conferma della diagnosi e del tipo di amiloidosi (AL vs ATTR), si dovrà necessariamente procedere ad un approfondimento diagnostico mediante biopsia endomiocardiaca utilizzando la tecnica dell'Immunogold o l'analisi proteomica (tabella 2). ${ }^{2,10}$

Entrambe le metodiche non sono di facile impiego e sono di pertinenza di pochi laboratori specializzati, come ad esempio quello del Centro di Riferimento nazionale per l'amiloidosi di Pavia.

Una volta esclusa una forma AL, e con essa il rischio di una misdiagnosi di tipo, si procederà a distinguere tra forma mutata e forma wt mediante l'analisi genetica; dopodichè il paziente potrà iniziare il suo corretto iter terapeutico.

\section{Evoluzione e prognosi}

Nelle forme mutate, l'evoluzione naturale dipende in larga misura dal tipo di mutazione e quindi dalla presenza di un fenotipo prevalentemente neurologico o cardiologico.

Nel primo caso la neuropatia periferica, anche in virtù dell'area geografica di appartenenza, può evolvere più o meno rapidamente in senso prossimale con il dolore e le alterazioni della sensibilità che interessano cosce e braccia, per manifestare poi un danno motorio con progressiva difficoltà alla deambulazione e all'uso dei movimenti fini delle mani, un interessamento vegetativo con alterazioni della funzionalità gastrointestinale, perdita di peso e cachessia. La mediana di sopravvivenza delle forme neurologiche varia dai 5 ai 15 anni. Tra le forme a prevalente o isolato interessamento cardiaco, siano esse wt o mutate (tra cui la Val122Ile è in assoluto la forma più diffusa), l'evoluzione naturale comporta un progressivo ispessimento delle pareti ventricolari con associati disturbi del ritmo, disfunzione diastolica e, infine, insufficienza ventricolare irreversibile. Le forme senili (wt) mostrano in genere una progressione più lenta della cardiopatia rispetto alle forme mutate, con una sopravvivenza mediana di circa 43 mesi vs i 25 mesi delle ATTRm. Quest'ultime mostrano comunque una sopravvivenza maggiore rispetto alle AL (figura 4).

La sopravvivenza mediana delle forme mutate aumenta tuttavia se si escludono le forme non-Val30Met e in particolare quelle ad esclusivo interessamento cardiaco, come la Val122Ile. Altri fattori che influenzano la prognosi sono la concentrazione di NT-proBNP e il grado di scompenso (classe NYHA). ${ }^{6}$

\section{Terapia}

\section{Terapia sintomatica}

La terapia sintomatica ha l'obiettivo di sostenere la funzionalità degli organi colpiti dalla malattia allo scopo di 
Tabella 2. Algoritmo semplificato in caso di sospetta amiloidosi cardiaca.

Percorso diagnostico sulla base del diverso grado di captazione cardiaca alla scintigrafia con difosfonati* e della presenza o meno di una componente monoclonale (CM).

Una forte captazione in assenza di CM è sufficiente per porre diagnosi di Amiloidosi cardiaca da TTR. Il test genetico indicherà poi se si tratta di una forma mutata o wt (senile). Se invece siamo in presenza di una captazione debole, indipendentemente dalla presenza o meno di una CM, si dovrà procedure obbligatoriamente ad un esame istologico mediante immunomicroscopia elettronica o spettroscopia di massa per distinguere tra una possibile forma AL o una da TTR.Nel caso di captazione assente, ma in presenza di una CM, dovremo escludere una forma AL mediante un prelievo di GPO o una biopsia endomiocardica. In assenza di CM potremo ragionevolmente concludere per una "non amiloidosi".

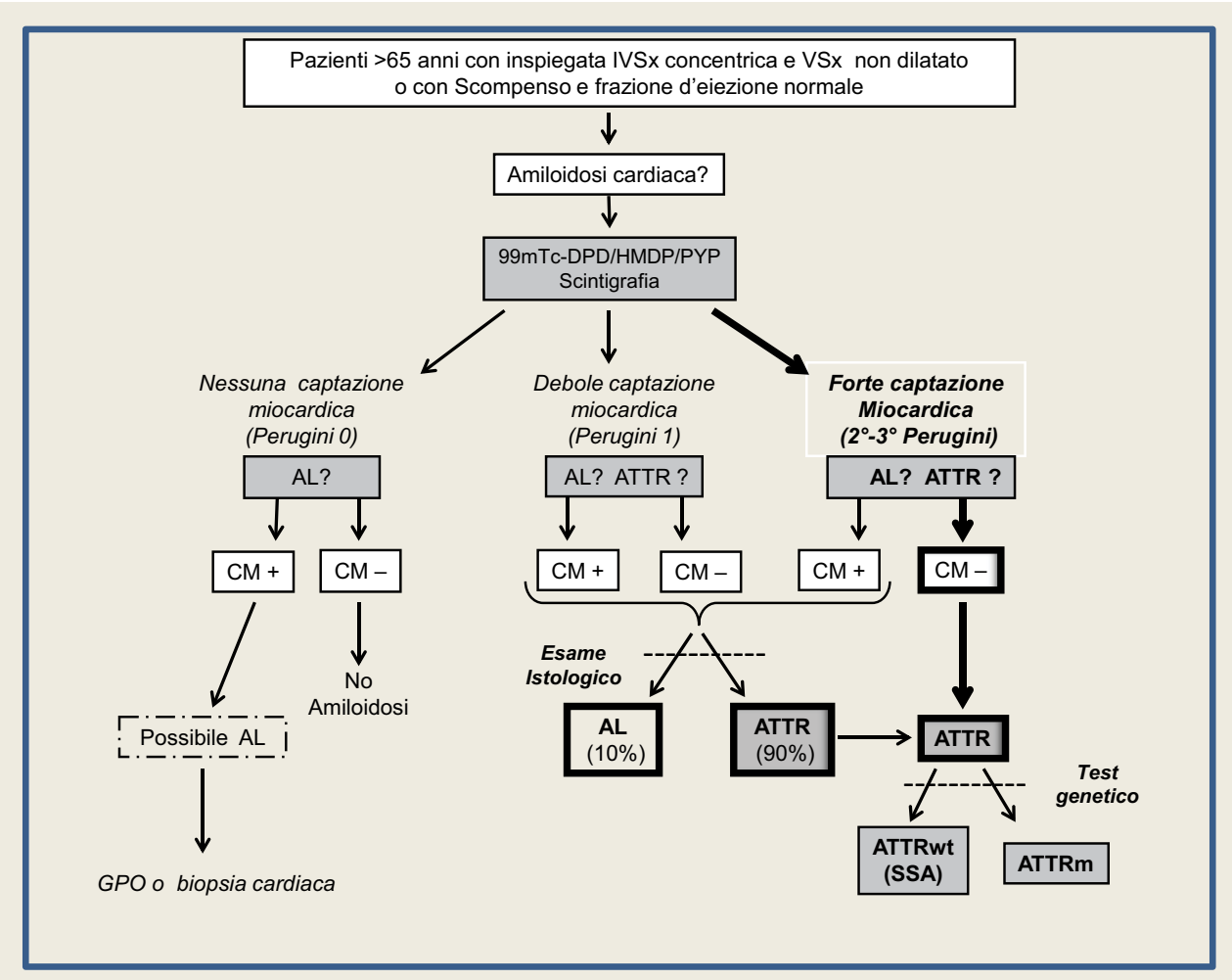

*Score Perugini: captazione forte (2-3), debole (I), assente (0); SSA: Amiloidosi Senile Sistemica.

mantenere una qualità di vita soddisfacente che consenta di attendere i risultati di eventuali terapie più mirate e specifiche volte a rallentare o invertire l'evoluzione della malattia. Questa comprende strategie dirette a controllare la sintomatologia dolorosa della polineuropatia (analgesici), gli eventuali disturbi autonomci (antidiarroici ecc...), i disturbi oculari (glaucoma e opacità vitreali) e quelli cardiaci.

Tra questi ultimi, i diuretici rivestono un ruolo centrale, anche se il loro uso deve essere monitorato con attenzione per evitare che un'eccessiva riduzione del precarico in pazienti con una patologia restrittiva comporti un calo eccessivo del riempimento ventricolare con conseguente riduzione della portata cardiaca ed ipotensione. L'impiego della terapia anticoagulante è d'obbligo nei pazienti che presentano flutter o fibrillazione atriale parossistica o permanente ed è da considerarsi anche in quei pazienti con un ritmo sinusale e documentata disfunzione atriale all' ecocardiogramma. ${ }^{8}$
È controindicato sia l'uso della digossina che, legandosi alle fibrille, si accumula e rischia di causare effetti tossici, che dei calcio-antagonisti per il loro effetto inotropo negativo. L'uso dei beta-bloccanti è ammesso, ma con cautela, per il rischio di ridurre la portata cardiaca. Analogamente, l'uso degli Ace-inibitori è ammesso, ma con cautela, per il rischio di indurre o aggravare gli episodi di ipotensione. L'utilità di impiantare un defibrillatore per la prevenzione della morte improvvisa resta poco chiara, poiché quest'ultima, nell'amiloidosi cardiaca, è principalmente correlata al verificarsi di una dissociazione elettromeccanica. ${ }^{8}$

\section{Terapia specifica}

Trapianto di fegato. È riservato alle sole forme mutate.

Il razionale è quello di rimuovere la principale fonte di produzione della TTR mutata presente in circolo 


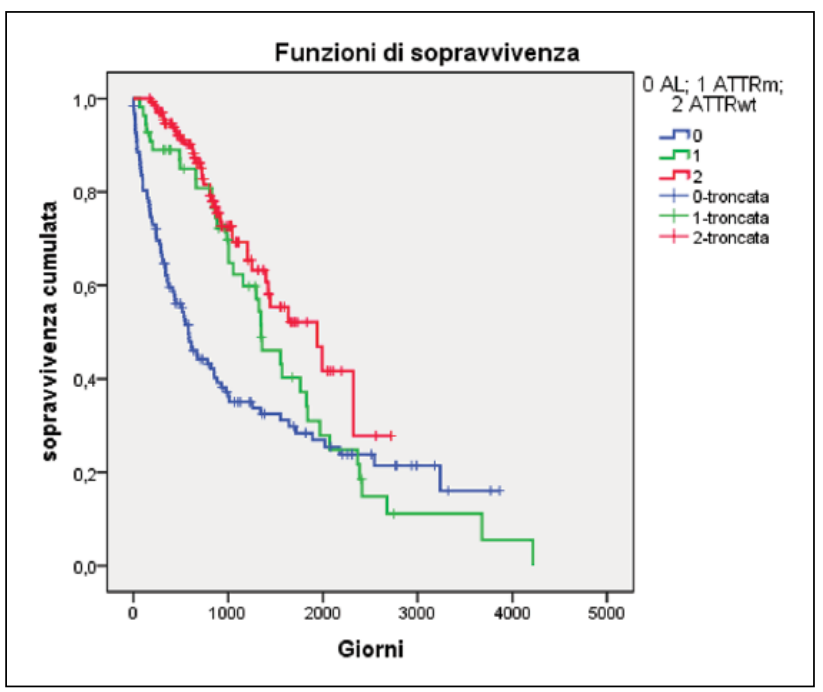

Figura 4. Curve di sopravvivenza (Kaplan-MeierM.) di pazienti con amiloidosi da TTR (mutata, $n=55 ; w t, n=129$ ) vs pazienti con amiloidosi $\mathrm{AL}(\mathrm{n}=123)$ reclutati presso il Centro di Riferimento Toscano per le Amiloidosi.

sostituendola con la TTRwt del donatore. Per questi pazienti, si ricorre al trapianto ortotopico di fegato che consiste nel trapiantare ad un paziente con TTR mutata il fegato di un donatore sano per trapiantare poi quello del paziente in un altro ricevente. 6,8

La sopravvivenza è dell' $82 \%$ a 5 anni nei pazienti con Val30Met, e del 59\% nei pazienti non-Val30Met.

I migliori risultati si osservano nei pazienti con Val30Met in fase iniziale di malattia, nei quali il trapianto sembra prevenire la progressione della neuropatia. In generale, i risultati del trapianto sono influenzati da vari fattori tra cui l'età, la gravità del danno neurologico e cardiaco al momento dell'intervento e il tipo di mutazione. Tuttavia, il trapianto non sembra risolvere del tutto il problema poiché in molti casi, soprattutto nei pazienti nonVal30Met, è stata osservata una progressione della malattia dovuta al depositarsi della TTR non mutata in quei tessuti, soprattutto quello cardiaco, dove erano già presenti depositi di amiloide prima del trapianto. ${ }^{6,8}$ Ciò sarebbe attribuibile ad un effetto di attrazione esercitato sulla molecola di TTRwt dalle fibrille amiloidi già depositate.

Il trapianto non previene la progressione dell'eventuale danno oculare o di quello a carico del SNC.

Il trapianto combinato di fegato e cuore può essere proposto nei casi di avanzato danno cardiaco.

Risultati incoraggianti con una buona sopravvivenza a lungo termine sono anche stati riportati dopo trapianto isolato cardiaco in pazienti con forme wt o mutate ad esclusivo coinvolgimento cardiaco. ${ }^{6}$

\section{Le nuove terapie}

Stabilizzatori della molecola della TTR. Gli stabilizzatori della molecola della TTR sono agenti in grado di stabilizzare la molecola della TTR nella forma tetramerica con cui circola nel sangue, prevenendo la sua dissociazione e successiva aggregazione in fibrille amiloidi.

Il Diflunisal e il Tafamidis sono due agenti recentemente utilizzati a questo scopo. Entrambi sono piccole molecole in grado di interagire con i siti di legame della TTR per la molecola della tiroxina, legandosi ad essi e stabilizzando la molecola prevenendone così la frammentazione e la successiva aggregazione in fibrille amiloidi.

Diflunisal è un agente antinfiammatorio non-steroideo (NSAID), non ufficialmente autorizzato per la terapia della ATTR, ma comunque utilizzato off-label al dosaggio di 250 $\mathrm{mg}$ due volte al dì per le sue intrinseche capacità di legarsi ai siti della tiroxina e dunque di stabilizzare la molecola della TTR. Gli studi condotti su pazienti affetti da ATTRm, con e senza V30M e con un grado di neuropatia variabile, ne hanno evidenziato l'efficacia pur in presenza di effetti collaterali prevalenti sull'apparato gastrointestinale e renale. I risultati sulle manifestazioni cardiache non sembrano invece altrettanto incoraggianti, fatta eccezione per alcuni dati riportati in un numero molto limitato di pazienti. ${ }^{6,8}$

Tafamidis è invece una piccola molecola appositamente sintetizzata allo scopo di stabilizzare il tetramero della TTR, ed è stato approvato in Europa alla dose di $20 \mathrm{mg} / \mathrm{die}$ per le forme neuropatiche (FAP) dei pazienti con V30M in stadio precoce dove si è dimostrato efficace nel rallentare la progressione della malattia. Meno efficace sarebbe risultato invece nelle fasi più avanzate per le quali non è prevista la rimborsabilità da parte del SSN. Il Tafamidis sembra ridurre la progressione della nefropatia in quei pazienti con Val30Met e coinvolgimento renale. ${ }^{8}$

Risultati incoraggianti sarebbero stati osservati anche nelle mutazioni non-Val30Met.

Recentemente la conclusione dello studio prospettico randomizzato a doppio cieco per l'impiego del Tafamidis a due dosaggi $(20 \mathrm{mg}$ e $80 \mathrm{mg}$ ) nel trattamento delle forme cardiopatiche sia mutate che wt in classe NYHA 1-3 ha per la prima volta dimostrato l'efficacia del farmaco nel ridurre la mortalità per tutte le cause e il ricorso all'ospedalizzazione per cause cardiovascolari. ${ }^{8,11}$ Il farmaco dovrebbe essere disponibile a breve per il trattamento dei pazienti affetti da cardiomiopatia da TTR sia mutata che wt.

Rimozione dei depositi di amiloide dai tessuti. Un'altra opzione terapeutica è quella di favorire la rimozione dei depositi di amiloide mediante farmaci come la Doxiciclina e l'Acido Tauroursodesossicolico (TUDCA) che sembrano avere un'azione sinergica. La prima sarebbe capace di destrutturare le fibrille facilitando i meccanismi endogeni di riassorbimento, mentre il secondo ridurrebbe la tossicità degli aggregati prefibrillari senza tuttavia avere un effetto sulle fibrille mature. I risultati dopo 12 mesi di trattamento hanno evidenziato la stabilità delle condizioni neurologiche e cardiologiche rispettivamente nel $46 \%$ e nel $75 \%$ dei pazienti trattati. ${ }^{6}$ 
Un altro possibile meccanismo di rimozione sfrutta l'attivazione complemento-indotta della degradazione dei depositi causata dall'impiego di anticorpi monoclonali rivolti contro la SAP (proteina presente in tutti i depositi di amiloide che stabilizza le fibrille e ne previene la rimozione) o contro altre componenti presenti nei depositi. 6,8

Blocco della sintesi epatica di TTR. Il blocco della sintesi di TTR a livello epatico può essere raggiunto interferendo o bloccando l'RNA messaggero della TTR, ovvero evitando che questo venga tradotto nella proteina corrispondente all'interno dei ribosomi. Due tipi di agenti sono attualmente in fase di studio: gli small interfering RNA (siRNA) e gli oligonucleotidi antisenso (ASO).

Questa strategia terapeutica è in grado di prevenire la produzione sia di TTRwt sia di TTRm, ed essere quindi un potenziale trattamento per entrambi i tipi di amiloidosi TTR-relata. ${ }^{12}$

\section{siRNA}

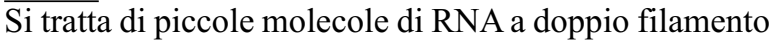
in grado di interferire con una sequenza della molecola del mRNA della TTR, sia wt che mutata, bloccandone la sintesi e provocandone la degradazione con una sensibile riduzione delle concentrazioni di TTR circolante.

\section{ASO}

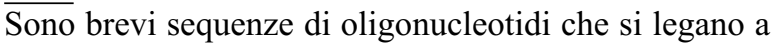
porzioni specifiche della molecola dell'mRNA della TTR provocando l'attivazione in loco della ribonucleasi $\mathrm{H}$, risultando nella degradazione della molecola di mRNA e riduzione della concentrazione plasmatica di TTR. Sono stati pubblicati recentemente $\mathrm{i}$ risultati di due studi clinici, l'uno impiegando Patisiran (siRNA) e l'altro Inotersen (ASO), che hanno dimostrato l'efficacia di entrambi i trattamenti nel rallentare la progressione delle manifestazioni neurologiche e nel migliorare la qualità di vita. Un miglioramento delle condizioni cardiache valutate sia con esame ecocardiografico sia mediante il rilevamento di una riduzione dei valori di NT-proBNP è stato riportato con l'uso del Patisiran. I due prodotti sono somministrati l'uno per via endovenosa e l'altro per via sottocutanea e sembrano ben tollerati.

\section{Conclusioni}

L'ATTR, nella sua variegata presentazione clinica, rappresenta oggi un'importante sfida diagnostica per l'internista, il cardiologo e il neurologo. La polineuropatia da un lato e la cardiopatia dall'altro, con cui possono esordire le forme mutate e per la seconda le forme wt, possono essere facilmente scambiate per altre patologie e ritardare di molti anni la diagnosi. Nel caso poi della presenza di una $\mathrm{CM}$, spesso incidentale nei pazienti anziani, la diagnosi può venire erroneamente attribuita ad una forma AL. Pazienti con una neuropatia periferica progressiva di origine sconosciuta, specialmente quelli con sintomi disautonomici, dovrebbero essere fortemente sospettati, così come quei pazienti cardiopatici con scompenso cardiaco e frazione di eiezione conservata.

La possibilità, oggi, di impiegare terapie farmacologiche mirate a colpire differenti bersagli lungo il processo di formazione delle fibrille amiloidi ("multi-target" therapy) aumenta considerevolmente le nostre capacità terapeutiche e rende ragione della sempre maggiore importanza di una diagnosi precoce.

\section{Dichiarazione di assenza di conflitto di interessi}

Gli Autori dichiarano di non avere conflitti di interessi.

\section{Finanziamenti}

Gli Autori dichiarano di non aver ricevuto finanziamenti specifici da qualsiasi ente nei settori pubblico, privato o senza fini di lucro.

\section{Bibliografia}

1. Rapezzi C, Quarta CC, Obici L, Perfetto F, et al. Disease profile and differential diagnosis of hereditary transthyretin-related amyloidosis with exclusively cardiac phenotype: an Italian perspective. European Heart Journal 2013; 34:520-528.

2. Amyloidosis: diagnosis and treatment. Gertz MA and Rajkumar SV eds. Springer, Berlin, Germany, 2010.

3. Hawkings PN, Ando Y, Dispenzieri A, et al. Evolving landscape in the management of transthyretin amyloidosis. Annals of Medicine 2015; 47:625-638.

4. Lachmann HJ, Booth DR, Booth SE, et al. Misdiagnosis of hereditary amyloidosis as AL (primary) amyloidosis. $N$ Engl JMed 2002; 346(23):1786-91.

5. Gillmore JD, Maurer MS, Falk RH, et al. Nonbiopsy diagnosis of cardiac transthyrethin amyloidosis. Circulation 2016; 133(24):2404-12.

6. Rapezzi C, Quarta CC, Riva L, Longhi S, et al. Transthyretinrelated amyloidosis and the heart: a clinical overview. Nat Rev Cardiol 2010; 7:398-408.

7. Gonzalez-Lopez E, Gallego-Delgado M, Guzzo-Merello G, et al. Wild-type transthyretin amyloidosis as a cause of heart failure with preserved ejection fraction. European Heart Journal 2015; 36:2585-2594.

8. Longhi S, Gagliardi G, Milandri A, Manuzzi L and Rapezzi C. La cardiopatia amiloidotica correlata alla transtiretina: alla ricerca del trattamento eziologico. G Ital Cardiol 2014; 15(5):293-300.

9. Lobato L and Rocha A. Transthyretin amyloidosis and the kidney. Clin J Am Soc Nephrol 2012, 7:1-10.

10. Farsetti S, Ciciani AM, Curciarello G, et al. Amiloidosi, il percorso diagnostico. Quando pensarci e come trovarla. $G$ Tec Nefrol Dial (in press).

11. Maurer MS, Schwartz J H, Gundapaneni B, et al. Tafamidis treatment for patients with transthyretin amyloid cardiomyopathy. N Engl J Med 2018; 379(11):1007-1016.

12. Buxbaum JN. Oligonucleotide drugs for transthyretin amyloidosis. N Engl J Med 2018; 379(1)82-85. 